\title{
18 Models for host-macroparasite interactions in micromammals
}

Rosà Roberto, Rizzoli Annapaola, Ferrari Nicola and Pugliese Andrea

\section{Introduction}

Modelling the spread of infectious diseases has proved to be useful for elucidating the conditions necessary for parasite persistence, the role of disease in population regulation and the expected patterns of host-pathogen coevolution.

Initially, models were applied to human infections (see, for instance, Bailey 1975). Anderson and May (1978) were among the first to use this type of model for natural animal populations, introducing also a different formulation for micro- and macro-parasites, to accommodate their diverse biological and epidemiological features. Microparasites characteristically increase rapidly in number when introduced into a susceptible host, and the precise count of infective agents is not only difficult to estimate, but irrelevant. In this case compartmental models are traditionally used that classify individuals in the population as either susceptible or infected or immune.

Reproduction in macroparasites usually includes the production of freeliving stages that pass from one host to the next after being exposed to a series of environmental constraints. Direct reproduction rarely occurs within the definitive host, although asexual reproduction can occur in the intermediate hosts of the digenean trematodes and some cestodes. Compared to microparasites, they are relatively large, have long generation times and are immunologically characterised by a diversity of antigens. Infections tend to be chronic, leading to morbidity rather than mortality, the severity of which tends to increase with the number of parasites harboured. Therefore, in order to understand epidemiological patterns in macroparasites, it is important to measure and consider not only the prevalence of infection (i.e. the proportion of infected hosts), but also the mean parasite 
burden as well as the entire distribution of parasite among host, since fertility, mortality and behaviour of the host population will depend on how parasites are distributed within it (Hudson and Dobson 1995).

An important feature of macroparasite infections is their aggregation within host populations. In human communities, for example, less than $20 \%$ of individuals generally harbour $80 \%$ of the helminth parasites present in that population. Thus, a relatively small number of individuals in the "tail" of the parasite distribution are responsible for the majority of parasite transmission and play an essential role in the persistence of the parasite (Anderson and May 1985; Woolhouse et al. 1997). This pattern has also been recently observed for the tick Ixodes ricinus and one of its rodent hosts, the yellow-necked mouse, Apodemus flavicollis (Perkins et al. 2003). Heterogeneities such as these are generated by variation between individual hosts in exposure to parasite infective stages, and by differences in susceptibility once an infectious agent has been encountered, which in turn depend on a series of intrinsic and extrinsic factors. A detailed review is presented by Wilson et al. (2002).

Host age is among the most important factors affecting parasite burden. In many host-parasite systems, especially those including rodent hosts, an increase in parasite abundance with age is reported (Abu-Madi et al. 1998; Behnke et al. 1999), although other patterns are possible. Hudson and Dobson (1995) classify the shape of the age-intensity curves as Type I (if parasite burden increases monotonically with age), Type II (if parasite burden reaches an asymptote), and Type III (if the parasite burden peaks at an intermediate age). Patterns of Types II and III have also been observed in populations of yellow-necked mice (A. flavicollis) and woodmice (Apodemus sylvaticus) (Quinnell 1992; Gregory et al. 1992).

There are a number of mechanisms that may affect age-intensity curves. These include parasite-induced host mortality, acquired immunity, agerelated changes in predisposition to infection, age-dependent changes in exposure, and age-related probabilities of accurately determining parasite loads (Hudson and Dobson 1995; Wilson et al. 2002). It is believed that acquired immunity develops in response to accumulated experience of infection and acts to decrease parasite establishment, survival, reproduction and/or maturation. Thus, in populations where transmission rates are high, the level of parasitic infection will rise rapidly, followed by a rapid increase in the level of acquired immunity causing a subsequent decline in parasite loads. In contrast, in populations where parasite transmission rates are low, parasite loads (and acquired immunity) will increase at a slower rate, and the age at peak infection will be greater. This will result in a negative correlation between peak levels of infection and the age at which 
the peak occurs, a phenomenon known as the "peak shift" (Anderson and May 1985). Peak shift has now been demonstrated in a number of human helminth infections, in several experimental infections of laboratory mice (Wilson et al. 2002), and in wild rabbit populations (Cattadori et al. 2005).

Host gender also has significant effects on parasite infection patterns. Epidemiologists have long recognized that males of vertebrate species, including humans, tend to exhibit higher rates of parasitism and disease than females (Wilson et al. 2002; Skorping and Jensen 2004). Sex biases in parasitism rates may be caused by physiological differences between males and females, such as in the levels of a number of steroid hormones, including testosterone, progesterone and estrogens. All of these hormones are known to have direct or indirect effects on components of the immune system and/or on parasite growth and development. Other mechanisms generating sex bias include differences in behaviour, diet composition and body size. In mammals, males are generally larger than females and there is good evidence that parasite load correlates with host size in a number of systems (Arneberg et al. 1998). Each sex may play a different role in the dynamics of parasitism, even if sex bias in parasitism rates does not occur; the example of A. flavicollis is discussed at end of sub-chapter 2 .

In sub-chapter 2, we review the main results emerging from models of infections with helminths having direct life-cycles. Reviews focusing on nematode parasites can be found in Roberts et al. (1995), Hudson et al. (2002) and Cornell (2005). Host-macroparasite interaction models were first applied to rodent hosts by Scott (1990), who combined theoretical and experimental studies of the dynamics of a laboratory mouse population infected with nematodes (Heligmosomoides polygyrus): at the end of subchapter 2 , we discuss the application of this type of model to a wild population of A. flavicollis.

Models of ectoparasite population dynamics seem to be less widespread, and are often motivated by an interest in the diseases that these parasites can transmit. For example, several models have recently been developed for tick-borne diseases, such as Lyme disease and tick-borne encephalitis (TBE), since these have become problematic in human populations of more temperate regions. Tick-borne disease systems are highly complex due to the presence of a number of heterogeneities coupled with non-linear phenomena operating in the transmission processes between tick, host and pathogen (Randolph et al. 2002). In sub-chapter 3, first we discuss models of ectoparasite population dynamics, in particular, tick dynamics; then, we briefly consider the tick-borne infection models that include rodent species as the principal pathogen reservoirs. 


\section{Models for rodent-nematode interactions}

\subsection{Models of nematode infection}

\subsubsection{Aggregation in parasite distribution}

In order to model macroparasitic infections it is crucial to quantify the parasite burden in the host population, since host mortality and morbidity depend on the number of parasites harboured. As well as prevalence (the proportion of hosts carrying at least one parasite), and mean parasite burden, it is also important to quantify the overall parasite distribution among hosts.

A key feature of macroparasitic infections is the aggregated distribution of parasites among hosts, meaning that most parasites are concentrated in a small number of hosts. Parasite distributions have traditionally been fitted with a negative binomial (Crofton 1971), for which the parameter $k$ is considered an index of aggregation. $k$ is relatively easy to estimate empirically: the closer $k$ is to 0 , the more aggregated the distribution; in contrast, when $k$ goes to infinity, the distribution approaches a Poisson. More recently, the Weibull distribution has been used as an alternative to the negative binomial and in some cases seems to be a more appropriate fit to macroparasite distributions (Gaba et al. 2005).

The causes of aggregation and its consequences on the dynamics of host-parasite interactions are still hotly debated. Several factors are thought to contribute to aggregation, including heterogeneities in hosts, predisposition to infection (Anderson and May 1985; Wilson et al. 2002), multiple infection (Isham 1995; Quinnel et al. 1995; Rosà and Pugliese 2002) and immunoepidemiological interactions (Grenfell et al. 1995). Several of these will be discussed briefly in the models below. The consequences of aggregation on the dynamics of host-parasite interactions are one of the main focus of the present chapter.

\subsubsection{The Anderson and May model}

In a seminal series of papers, Anderson and May (1978) modelled the effect of aggregation by assuming that, regardless of the causes, parasite distribution can be represented by a negative binomial, with a fixed aggregation parameter, $k$, and by studying the effect of $k$ on parasite dynamics. In their model, the distribution of adult macroparasites is described entirely 
by the variable $x(t)$, representing the mean parasite burden, and the constant $k$ (estimated in the field). All free-living stages are usually grouped into a single stage, $L(t)$, and any time delay between egg release and development of infectious stages is neglected. Hence the variables of the model are $x(t)$, the mean parasite burden, $L(t)$, the density of free-living larvae, and $N(t)$, the density of hosts. The resulting system is:

$$
\begin{aligned}
& \frac{d N}{d t}=N\left((b-d)\left(-\frac{N}{K}\right)-b\left(1-\left(\frac{1}{1+\frac{\xi}{k} x}\right)^{k}\right)-\alpha x\right) \\
& \frac{d x}{d t}=\beta \psi L-x\left(b\left(\frac{1}{1+\frac{\xi}{k} x}\right)^{k}+\sigma+\alpha+\frac{\alpha}{k} x\right) \\
& \frac{d L}{d t}=h N x-\delta L-\beta L N .
\end{aligned}
$$

The equations in system (1) are based on very simple assumptions: hosts are born and die according to a logistic demographic model ( $b$ and $d$ are birth and death rates; $K$ is the carrying capacity). Moreover, if a host carries $i$ parasites, its death rate increases linearly with $i$ ( $\alpha$ is the proportionality constant) and its birth rate decreases multiplicatively ( $\xi$ measures the intensity of decrease). Adult parasites die at rate $\sigma$ and give birth to larvae at rate $h$. Finally, larvae die at rate $\delta$ and randomly encounter hosts and are ingested at rate $\beta ; \psi$ is the probability that they then develop into adult parasites. However, the translation of these assumptions about individual hosts and parasites into equations for aggregated variables, such as $x(t)$, is not obvious. In fact, system (1) can be considered a type of moment closure of an infinite system, whose variables are $p_{i}(t)$, the number of hosts carrying $i$ adult parasites at time $t$, first proposed by Kostizin (1934) and rather difficult to study (see Pugliese and Tonetto 2004 for a recent rigorous treatment). Note that the term in brackets describing parasite-induced reduction in fertility is rather complex, and is different from the simple linear term present in Anderson and May (1978). Indeed, their linearity assumption is untenable, and the term considered here is due to work by Diekmann and Kretzschmar (1991); however, in the linear approximation it is the same, and indeed it is simply equal to 0 when $\xi=0$ (the assumption used in the case study found in sub-chapter 2). 


\subsubsection{Qualitative predictions of the model}

One of the most important contributions of modelling host-pathogen interactions has been introduction of the concept of the basic reproduction number $\left(R_{0}\right) . R_{0}$ is a quantity that describes the average number of infective agents produced by one infective agent in the initial phase; if $R_{0}>1$ a parasite species can invade a host population, and persist in it. This concept can also be used for macroparasites, and $R_{0}$ can be computed for model (1) as

$$
R_{0}=\frac{\psi \beta K}{\delta+\beta K} \frac{h}{b+\sigma+\alpha}
$$

The first factor in (2) expresses the probability that a larva finds a host and develops into an adult; the second factor, the average number of larvae produced by an adult parasite during its lifetime. From the expression (2) one can see that successful parasites will have a high fecundity $(h)$ and a small effect on host mortality $(\alpha)$, which is the basis of theories on parasite evolution (Anderson and May 1982; Pugliese 2002). On the other hand, it can be seen that, for model (1), there is no effect on $R_{0}$ of the aggregation $k$, and of the parasite-induced fertility decrease $\xi$.

When $R_{0}>1$, system (1) reaches an equilibrium where parasites are at a positive level, and the host population is below carrying capacity, and thus, being regulated by parasites. An analysis of the model clearly shows that a host population can be regulated by a parasite to a density significantly lower than the carrying capacity, even though parasite density is low and parasite-related deaths are rare.

It is also possible to analyse the stability of the equilibrium where hosts and parasites coexist. In an ideal case, with Malthusian host demography, no effect of parasites on host mortality or fertility, infinitely quick larval dynamics, and no parasite aggregation, there would be a continuum of neutrally stable equilibria. Against this neutral baseline, all factors can be seen as stabilizing or destabilizing: host logistic demography, parasite-induced host mortality, and parasite aggregation are stabilizing factors, whereas parasite-reduced host fertility and long-lived larvae are destabilizing. If only stabilizing factors are present in the model, host-parasite dynamics will tend towards a unique equilibrium; if only destabilizing factors are included, the equilibrium is unstable and solutions will tend to periodic cycles; if both stabilizing and destabilizing factors are present, the result will depend on the relative strength of the contrasting factors.

A particularly good example of this approach can be found in Dobson and Hudson (1992) on the population cycles of red grouse (Lagopus lagopus scoticus). They showed that many patterns of population cycles 
were in accordance with model predictions, and were also able to confirm experimentally the role of parasites in maintaining the cycles (Hudson et al. 1998).

\subsubsection{Extensions of the model}

The Anderson and May model is based on an empirical moment closure; however, it is obviously possible to consider other approximations. Such a model was proposed by Adler and Kretschmar (1992) to enable the degree of aggregation to evolve dynamically. Other moment closure methods have also been developed, such as the normal approximations used in a stochastic setting by Herbert and Isham (2000).

More interestingly, it is possible to extend model (1) by considering other phenomena such as multiple infections and host heterogeneity, age structure and the dynamics of the immune response. For example, Rosà and Pugliese (2002) analysed models where aggregation in parasite distribution was generated by including multiple infections or host heterogeneity (due, for instance, to sex differences in susceptibility). They found that, with either mechanism, parasite aggregation always has a stabilizing effect; however, the quantitative strength of the effect is rather different depending on the biological mechanism that produces them.

It is well recognized that individual immune response has a strong influence on the dynamics of parasitism, and cannot be ignored, especially when analysing parasite aggregation. Most models describe immunological status as a single variable, $m$, varying according to the equation

$$
\frac{d m}{d t}=X-\mu_{I} m
$$

where $\mathrm{X}$ represents parasite abundance (larval challenge, adult burden, or another factor, according to the case study), and $\mu \mathrm{I}$ allows for finite immunological memory (Woolhouse 1992). It is extremely difficult to integrate such an equation for individual immunity at the population level (Grenfell et al., 1995). However, Roberts and Heesterbeek (1995) used (3) for measuring average immunity in a model of domestic animals, restocked every year.

\subsubsection{Age-related parasite intensity}

Individual immune response has been considered most often in models for explaining the age patterns of parasitism. In this case, individual parasite load is assumed to change with age according to some stochastic process. The simplest case is to assume constant rates of parasite acquisition and 
death, so that parasite load increases monotonically with age, and parasite distribution at any fixed age should be Poisson. Since these results are generally in contrast with observed patterns, more complex models have been developed that yield predictions in better agreement with data. In particular, Type III age intensity curves (see Introduction) seem to indicate a role for immune response in decreasing susceptibility to infection and increasing parasite death rates, although it is not easy to detect the role of the immune response in available data (Chan et al. 2000). However, clumped infections, and intrinsic differences in host susceptibility seem to be necessary to explain the relatively high aggregation found in parasite distributions, even when age and sex are discounted (Duerr et al. 2003).

As discussed above, it is very difficult to integrate these models at the level of an individual host with the models at the population level considered above. A simpler approach is to use a phenomenological model that assumes age difference in susceptibility (without attempting to derive them from a model of immunity development). Such an approach has been used by Rosà et al. (2000) to model the interaction of a chamois (Rupicapra rupicapra) population and Trichostrongildae parasites. The results of this study clearly demonstrate a difference between models that consider age differences in susceptibility and those that do not. All other factors being equal, the model including age differences predicts a higher density for the host population, a lower parasite load, and a more aggregated distribution; in addition, parasites are concentrated in fewer hosts, and have a lower effect on the host population.

\subsubsection{The stochastic approach}

Models based on differential equations are justified when populations are large, and can capture a relevant part of the phenomenology of population interactions. However, small populations (and related phenomena, such as local extinctions) can only be described by stochastic models; moreover, it is conceivable that a stochastic model in which interactions are modelled at the individual level yields different results than a deterministic model that is based on some sort of averaging. The recent, marked increase in computing power now makes it possible to run simulations of complex individual-based models with hundred of thousands of individuals, so that the predictions of deterministic models can be compared to outcomes of simulations.

For example, Rosà et al. (2003a) have analysed simulations of a stochastic model, based on the same assumptions as the deterministic models, and focusing on two aspects: population cycles and extinctions. Whereas deterministic models predict damped population cycles, stochastic simula- 
tions often exhibit reasonably regular and sustained cycles for the same parameter values, in agreement with the general idea that stochastic perturbations can sustain otherwise damped cycles (Kaitala et al. 1996). Population extinctions have been examined for parameter values suitable for the Ascaridia compar infection in rock partridge (Alectoris graeca saxatilis), a species disappearing from the Alps. It was found that, since partridge population were small, parasite populations could not persist on only a single host, but had to rely on additional hosts, such as the black grouse (Tetrao tetrix), that has a much higher population density. That a generalist parasite may drive to extinction a small host population is widely accepted (McCallum and Dobson 1995); therefore, an intriguing result of the simulation analysis of these host-parasite interactions is that, for intermediate population sizes, the extinction probability is more or less constant or even decreases with increasing external infection (that is, with an increase in the degree to which parasites are shared). In simulation models, many other factors may easily be integrated, from the dynamics of individual immune responses, to the genetic structure of populations, to behavioural or spatial differences among individuals (Hess 1996; Keeling 1999).

In conclusion, there is great potential for understanding the role of various factors in host-parasite dynamics, and consequently, for designing appropriate control measures. We firmly believe that simple deterministic models will provide useful qualitative predictions, while complex simulation models are best used for exploring the qualitative effect of more realistic assumptions, rather than as predictive tools.

\subsection{Case study: Apodemus flavicollis - Heligmosomoides polygyrus interactions}

Among the numerous macroparasites infecting micromammals, Heligmosomoides polygyrus is often investigated because of its high prevalence and abundance in wild populations. Furthermore, its biological and immunological features make it a good laboratory model for studying gastrointestinal parasite infections. H. polygyrus (Family Trichostrongylidae) has a direct life cycle which is completed in 13-15 days with some variation among subspecies (Keymer, 1985). Infection occurs by ingestion of the third stage of free-living larvae $\left(L_{3}\right)$ (Slater and Keymer 1988; Scott 1990). Once ingested, larvae colonize the small intestine and develop into adult parasites that live up to 3 months depending on the H. polygyrus strain and immunological status of the host (Gregory et al. 1990). Eggs produced by adult female worms are deposited in the host's faeces where hatch and develop to infective $L_{3}$ (Anderson 2000). $H$. polygyrus has a broad infective 
spectrum, with possible hosts including Apodemus spp., Mus musculus musculus, Mus musculus domesticus, and Peromiscus maniculatus, while its occurrence in two species of Clethrionomys spp. is debated (Lewis 1987; Behnke et al. 1991).

Studies of the effects of $H$. polygyrus on host population dynamics led to the first empirical evidence of the regulatory role of parasites on host populations confirming the theoretical predictions formulated by Anderson and May (1978). The discovery that inbred mice reared on damp peat maintained a self-sustained infection cycle of $H$. polygyrus without any artificial intervention provided the basis of a free-running experimental system for investigations on this host-parasite interaction. Scott (1987) placed three yellow-necked mouse populations on this type of arena, and by supplying a fixed amount of food, and created density dependence in host populations growth (Scott 1987). After an exponential increase, the mouse populations reached an equilibrium density of $320 \mathrm{mice} / \mathrm{m}^{2}$ (Scott 1987). Afterward $H$. polygyrus was introduced, the mice $10 \%$ of their density with respect to control arenas. Following pharmacological removal of the parasite from the populations, host density was restored to its original level. Scott (1990) then carried out a series of detailed analyses with the help of mathematical models, to identify the consequences of parasite infection on host mortality. The fact that the output of the model matched observed data confirmed the theoretical predictions that the main effect of $H$. polygyrus was reduction on mice survival while the effect on host fecundity seems to be negligible (Scott 1990). Subsequent research in seminatural populations in outdoor enclosures confirmed that the negative effect of $H$. polygyrus on mouse population growth was mainly a consequence of parasite induced mortality (Gregory 1991; Quinnell 1992).

Other studies on $H$. polygyrus focused on the mechanisms promoting the maintenance of infection. Sex bias in parasite load has been documented in most host-parasite systems and although factor generating this bias have been analysed, the consequences for parasite persistence have been poorly investigated (Poulin 1996; Schalk and Forbes 1997; McCurdy et al. 1998; Moore and Wilson 2002; Wilson et al. 2002). The Apodemus spp. $-H$. polygyrus systems are an exception to this rule, suggesting that both sexes contribute equally to parasite maintenance (Gregory 1992; Gregory et al. 1992; Abu-Madi et al. 1998; Behnke et al. 1999; Skorping and Jensen 2004). Experimental field manipulation of parasite load in male or female mice indicated that males play a major role in driving the infection in the whole host population: while removal of $H$. polygyrus from male mice resulted in a reduction in parasite load even in untreated hosts, removal of parasites from females had no effect on the parasite load of un- 
treated hosts. (Ferrari et al. 2004). Results of the field study did not allow the identification of the underlying parasitological mechanism involved, but mathematical modelling suggests the pattern was caused by sexual differences in immunological response or behaviour (according to spatial arrangement of free infective larvae). The following simple model considers a separate dynamics for male and female hosts; the equations are the following:

$$
\begin{aligned}
& \frac{d M}{d t}=b F+M\left[-d_{M}-\left(b-d_{M}\right)(M+F) / K-\alpha_{M} x_{M}\right] \\
& \frac{d F}{d t}=F\left[b-d_{F}-\left(b-d_{F}\right)(M+F) / K-\alpha_{F} x_{F}\right] \\
& \frac{d x_{M}}{d t}=x_{M}\left[-\sigma_{M}-b-\alpha_{M}\left(x_{M} / k_{M}+1\right)\right]+\beta_{M} \psi_{M} L \\
& \frac{d x_{F}}{d t}=x_{F}\left[-\sigma_{F}-b-\alpha_{F}\left(x_{F} / k_{F}+1\right)\right]+\beta_{F} \psi_{F} L \\
& \frac{d L}{d t}=h_{M} M x_{M}+h_{F} F x_{F}-\delta L-\beta_{M} M L-\beta_{M} F L,
\end{aligned}
$$

where $M$ and $\mathrm{F}$ are densities of male and female hosts, respectively, $x_{M}$ and $x_{F}$ are the average load of parasites harboured by males and females, respectively and $L$ represents the common free-living infective pool.

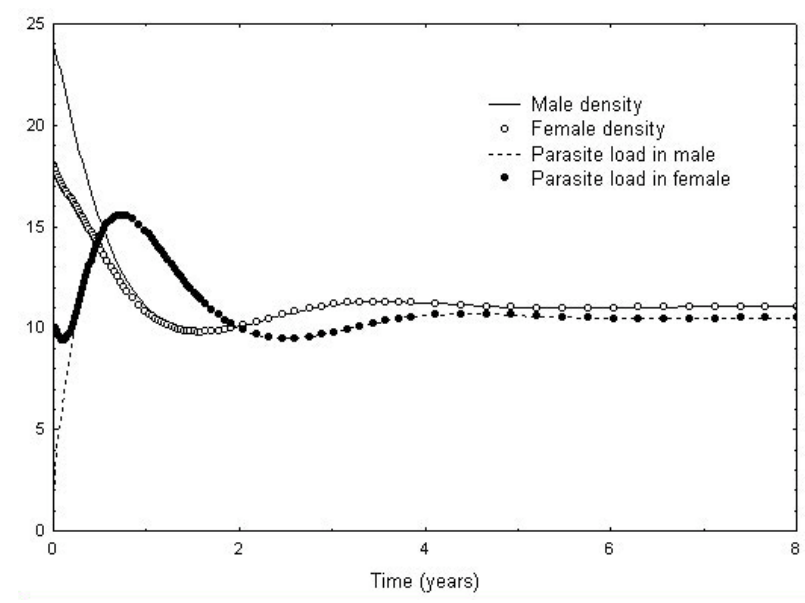

Fig. 1. Temporal dynamics of male and female A. flavicollis and their average load of $H$. polygyrus, for the case where fertility of parasites found on male hosts is greater than those found on female hosts $\left(h_{M}>h_{F}\right)$. Parameters values are those in Table 1 
Table 1. Numerical values of population parameters for A. flavicollis and H. polygyrus

\begin{tabular}{|c|c|c|c|}
\hline Symbol & Parameter & Value & References \\
\hline $\bar{d}$ & Host death rate & $3.7 \cdot 10^{-3}$ & Flowerdew, 1984 \\
\hline$b$ & Host birth rate & $8.21 \cdot 10^{-3}$ & Flowerdew, 1984 \\
\hline$K$ & $\begin{array}{l}\text { Host population carrying ca- } \\
\text { pacity }\end{array}$ & 50 & $\begin{array}{l}\text { Unpublished data on } \\
\text { A. flavicollis in Trentino }\end{array}$ \\
\hline$\sigma$ & $\begin{array}{l}\text { Mortality rate of adult para- } \\
\text { site }\end{array}$ & $1.3 \cdot 10^{-2}$ & Gregory et al. 1990 \\
\hline$\psi$ & $\begin{array}{l}\text { Proportion of ingested infec- } \\
\text { tive larvae that develop to } \\
\text { the adult stage }\end{array}$ & $8 \cdot 10^{-2}$ & $\begin{array}{l}\text { Slater and Keymer 1988; } \\
\text { Enriquez et al. } 1988 ; \\
\text { Gregory et al. } 1990\end{array}$ \\
\hline$\alpha$ & $\begin{array}{l}\text { Mortality rate of host due to } \\
\text { the parasite }\end{array}$ & $2.4 \cdot 10^{-4}$ & Keymer and Hiorns 1986 \\
\hline$K$ & $\begin{array}{l}\text { Aggregation parameter of the } \\
\text { negative binomial distribu- } \\
\text { tion }\end{array}$ & 0.36 & Ferrari et al. 2004 \\
\hline$\delta$ & $\begin{array}{l}\text { Mortality rate of free-living } \\
\text { infective stages }\end{array}$ & $1.6 \cdot 10^{-2}$ & $\begin{array}{l}\text { Dobson and Hudson 1992; } \\
\text { Fernàndez et al. } 2001\end{array}$ \\
\hline$h_{M}$ & $\begin{array}{l}\text { Rate of production of infec- } \\
\text { tive larvae per adult parasite } \\
\text { in male mice }\end{array}$ & 1.67 & $\begin{array}{l}\text { Based on observed data on } A \text {. } \\
\text { flavicollis in Trentino }\end{array}$ \\
\hline$h_{F}$ & $\begin{array}{l}\text { Rate of production of infec- } \\
\text { tive larvae per adult parasite } \\
\text { in female mice }\end{array}$ & 0.33 & $\begin{array}{l}\text { Based on observed data on } A \text {. } \\
\text { flavicollis in Trentino }\end{array}$ \\
\hline$\beta$ & $\begin{array}{l}\text { Rate of ingestion of free- } \\
\text { living larvae }\end{array}$ & $4 \cdot 10^{-4}$ & $\begin{array}{l}\text { Based on observed data on } A \text {. } \\
\text { flavicollis in Trentino }\end{array}$ \\
\hline
\end{tabular}

Fig. 1 shows a simulation obtained with model (4) under the assumption that parasite fertility $(h)$ is different for parasites harboured by hosts of a different sex. Specifically, eggs expelled by parasites in males have a higher hatching rate and larval survival than eggs expelled by parasites in females (i.e. $h_{M}>h_{F}$, Table 1). All other parameter values are the same for both sexes and are those reported in Table 1. A. flavicollis $-H$. polygyrus system reaches a stable equilibrium through damped oscillations, with no sex-bias in parasite burden and host density between the two host sexes (Fig. 1).

\section{Models for host-tick interactions}

Tick-borne infections transmitted by Ixodid ticks are increasing in many parts of Eurasia and North America. Several investigations of the epidemi- 
ology of tick-borne diseases have been performed in recent years, including that of Lyme disease, tick-borne encephalitis (TBE) and human granulocytic anaplasmosis (HGA). The increase in prevalence of these diseases is associated with different ecological and sociological changes such as the abandonment of fields and pastures coupled with the expansion of woodland, which favour an increase in the range and densities of suitable hosts for ticks; and hence, the potential for disease transmission by ticks.

The complexity of tick-borne infection dynamics has required the development of specific mathematical models. Clearly, these models require the description of tick population dynamics, reviewed by Kitron and Mannelli (1994). Models of tick population dynamics, as well as for tick-borne infections, can be classified as computer-based models, which often include many details of the interactions, and simple models, mainly suited for qualitative results. The latter generally assume continuous time, despite the fundamental importance of seasonality on tick dynamics in a temperate area. Hudson et al. (1995), Norman et al. (1999) and Rosà et al. (2003b) have developed different models with the specific aim of exploring the effect of host abundance and host community composition on persistence of ticks and pathogens. For example, detailed field investigations of Louping Ill disease coupled with large scale experimental studies showed that reducing the population size of some species can have a profound effect on the dynamics and persistence of infection in other species (Hudson et al. 1995; Gilbert et al. 2001). Other studies have focused instead on the deer tick-Borrelia system in northeastern America examining how the species richness of the host community influences the persistence of Lyme disease (Ostfeld and Keesing 2000; LoGiudice et al. 2003). These authors found that the percentage of nymphal ticks infected (and hence risk to humans) was dependent on the abundance of the white-footed mouse Peromyscus leucopus, relative to other non-rodent hosts. This suggests that the preservation of vertebrate biodiversity and community composition can reduce the incidence of Lyme disease (Lo Giudice et al. 2003).

Norman et al. (1999) computed $R_{0}$ for tick-borne infections and introduced the so-called dilution effect: i.e. when two alternative hosts exist for ticks, only one of which is competent for transmission, an increase in the density of the incompetent host may shift $R_{0}$ from above to below 1 , and thus cause pathogen extinction. Qualitatively similar results have been obtained in computer-based models (Van Buskirk and Ostfeld 1998). Rosà et al. (2003b) and Rosà and Pugliese (submitted) have extended the model by computing $R_{0}$ in several cases and exploring the dilution effect in greater detail. Randolph et al. $(1996,1999)$ have studied the effect of seasonality, and have obtained a rough estimate of $R_{0}$, for TBE. They found $R_{0}>1$ only 
where nymphs and larvae follow a similar pattern of emergence. Moreover, they related this pattern to climatic factors, and, using satellite data, showed that certain climatic patterns could explain the occurrence of TBE in many part of Europe (Randolph et al. 2000). In fact, the TBE virus only persists in those geographic areas where a combination of biotic and abiotic factors favours co-feeding of larvae and nymphs on rodent hosts, predominantly the yellow-necked mouse, permitting the non-systemic transmission of the virus (Labuda et al. 1993, 1997; Jones et al. 1997; Labuda and Randolph 1999; Randolph et al. 1999, 2000; Randolph 2001). This transmission mechanism affords a greater degree of virus amplification than the conventional viraemic route of transmission (Randolph et al. 1996, 1999, 2002). It appears that the co-feeding activity of larvae and nymphs occurs in areas where a rapid decrease in daily autumnal temperature (rapid autumnal cooling) delays the host-seeking activity of summerborn larvae until the following spring (Randolph 2001), when they then feed together with nymphs on hosts (Fig. 2).

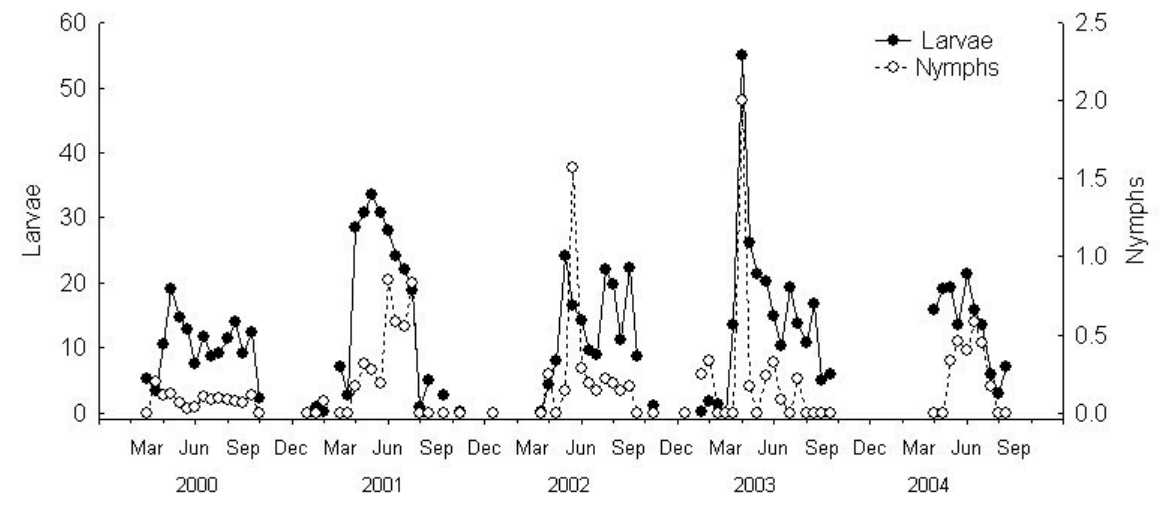

Fig. 2. Observed values of the average load of feeding larvae and nymphs on $A$. flavicollis during the period 2000-04 in a TBE endemic area in Trentino (northern Italy)

Given this dependence on climate, the current distribution of TBEV foci is expected to shift towards higher latitudes and altitudes over the next 50 years as the climate becomes warmer (Randolph and Rogers 2000; Randolph 2001; Zeman and Benes 2004).

A sophisticated tick population model that incorporates the effect of climate variables such as temperature in the demography of tick population was developed by Randolph and Rogers (1997) for the African tick Rhipicephalus appendiculatus. The model includes temperature- and den- 
sity-dependent rates together with climate-driven density-independent laws for different tick stages, and describes both the seasonality and the annual range of variation in numbers of each stage in different sites in Africa. The model is potentially applicable to other tick species for predicting tick abundance and seasonality as risk factor for tick-borne diseases.

Finally, more complex models use multi-dimensional state variables and ticks are usually classified not only on the basis of stages but also on age. The complexity of these models requires the application of appropriate mathematical techniques such as Leslie matrix models and dynamic life tables. For example, Mount and Haile $(1987,1989)$ developed computer simulation models for different American tick species. They built agespecific life-table tick population models, where each tick stage is divided into discrete age classes. Density-dependent and temperature-dependent constraints were included in these models and the growth and the generation time for tick populations were simulated for several input levels of day length, weather, habitat and host density.

In this sub-chapter, we discuss a simple model for tick population dynamics recently introduced by Rosà and Pugliese (submitted), then add the dynamics of the pathogen. Throughout, we focus on the effect of the relative abundance of the main host species on the persistence and the dynamics of tick populations and the pathogen.

\subsection{A simple tick population model}

The life cycle of Ixodid ticks includes three post embryonic developmental stages: larva $L$, nymph $N$ and adult $A$. Each stage can be subdivided in turn according to the phases of activity: "questing", in which the unfed tick seeks a host and 'feeding' in which the attached tick feeds, becomes engorged and drops off. After dropping off their hosts, ticks complete a period of development, after which they emerge as questing ticks at the next stage (or lay eggs, in the case of adult females). Ticks are found on many vertebrate hosts; usually adults have a more restricted host range than larvae and nymphs (Eisen and Lane 2002). Nevertheless, in many natural systems, the dynamics of ticks and tick-borne diseases (e.g. Lyme disease and TBE) depends largely on two classes of hosts: small rodents such as mice and voles and larger mammals, especially ungulates. Rodents, which will be indicated in the following model as $H_{1}$, are the most common host species for immature stages of ticks (larvae and nymphs) while adults are generally found on medium-sized and large mammals $\left(H_{2}\right)$, especially deer. 
A simple model for the dynamics of tick populations has been developed assuming that host populations are fixed at given densities $H_{1}$ and $H_{2}$. This model, as for most models of tick-borne infections, is continuous, i.e. it disregards seasonality. A different approach has been used by Ghosh and Pugliese (2004) which introduces seasonality through a semi-discrete model. The parameters included in the model are summarized in Table 2 and the resulting equations that describe the tick population dynamics are the following (Rosà and Pugliese, submitted):

$$
\begin{aligned}
& \frac{d L_{Q}}{d t}=\sigma^{A} a_{T}(T) A_{F}-d^{L} L_{Q}-\left(\beta_{1}^{L} H_{1}+\beta_{2}^{L} H_{2}\right) L_{Q} \\
& \frac{d L_{F}}{d t}=\left(\beta_{1}^{L} H_{1}+\beta_{2}^{L} H_{2}\right) L_{Q}-\sigma^{L} L_{F} \\
& \frac{d N_{Q}}{d t}=m^{L} \sigma^{L} L_{F}-d^{N} N_{Q}-\left(\beta_{1}^{N} H_{1}+\beta_{2}^{N} H_{2}\right) N_{Q} \\
& \frac{d L_{F}}{d t}=\left(\beta_{1}^{N} H_{1}+\beta_{2}^{N} H_{2}\right) N_{Q}-\sigma^{N} N_{F} \\
& \frac{d A_{Q}}{d t}=m^{N} \sigma^{N} N_{F}-d^{A} A_{Q}-\left(\beta_{1}^{A} H_{1}+\beta_{2}^{A} H_{2}\right) A_{Q} \\
& \frac{d A_{F}}{d t}=\left(\beta_{1}^{A} H_{1}+\beta_{2}^{A} H_{2}\right) A_{Q}-\sigma^{A} A_{F} .
\end{aligned}
$$

Encounters between questing ticks and hosts of either class are governed by mass-action; for example the corresponding encounter rate with questing nymphs is given by the product $\left(\beta_{1}^{N} H_{1}+\beta_{2}^{N} H_{2}\right) N_{Q}$. A tick-host encounter results in the transition of the tick to the feeding stage. Questing larvae, nymphs and adults die at rate $d^{L}, d^{N}$ and $d^{A}$, respectively (Table 2). Mortality in the feeding period, which lasts on average $1 / \sigma$ days, is neglected. The parameters $m^{L}$ and $m^{N}$ represent the probability of moulting success for larvae and nymphs after feeding, respectively. Finally, we assume that the production of larvae per feeding adult tick is densitydependent, and is represented by a decreasing function $a_{T}(\mathrm{~T})$; below, we consider different choices for this function.

Through the study of the local stability of the tick-free equilibrium (Rosà et al. 2003b) the following basic reproduction number for the tick population can be derived:

$$
R_{0, \text { icks }}=a_{T}(0) \frac{m^{L}\left(\beta_{1}^{L} H_{1}+\beta_{2}^{L} H_{2}\right)}{d^{L}+\beta_{1}^{L} H_{1}+\beta_{2}^{L} H_{2}} \frac{m^{N}\left(\beta_{1}^{N} H_{1}+\beta_{2}^{N} H_{2}\right)}{d^{N}+\beta_{1}^{N} H_{1}+\beta_{2}^{N} H_{2}} \frac{\beta_{1}^{A} H_{1}+\beta_{2}^{A} H_{2}}{d^{A}+\beta_{1}^{A} H_{1}+\beta_{2}^{A} H_{2}} .
$$


The quantity in (6) represents the threshold condition for the persistence of ticks in the system. When $R_{0, \text { ticks }}>1$ ticks will persist and tick and host populations will settle to a positive coexistence equilibrium. The quantity $R_{0, t i c k s}$ has a rather simple biological interpretation in that, if the product of the losses from each tick stage is greater than the product of the gains to each stage, then the ticks will die out, otherwise they will persist.

Table 2. Notation and values for variables and parameters included in the models

\begin{tabular}{ll}
\hline Symbol & Description \\
\hline Ticks and host densities \\
$L_{Q} \quad$ Density of questing larvae \\
$L_{F}$ & Density of feeding larvae \\
$N_{Q}$ & Density of questing nymphs \\
$N_{F}$ & Density of feeding nymphs \\
$A_{Q}$ & Density of questing adults \\
$A_{F}$ & Density of feeding adults \\
$T$ & Density of total tick population \\
$H_{1}$ & Density of hosts 1 (rodents) \\
$H_{2}$ & Density of hosts 2 (roe deer) \\
Demography and encounter parameters for ticks and hosts \\
$d^{i} \quad$ Natural death rate of hosts $H_{i}(i=1,2)$ \\
$d_{z} \quad$ Natural death rate of questing ticks in stage $z(z=L, N, A)$ \\
$a_{T} \quad$ Average number of larvae produced per fed adult tick \\
$\sigma^{z}$ & Detachment rate of feeding ticks in stage $z(z=L, N, A)$ \\
$M^{z}$ & Moulting success probability for ticks of stage $z(z=L, N)$ \\
$\beta_{i}^{z}$ & Encounter rates between questing ticks of stage $z(z=L, N, A)$ and hosts \\
Infection parameters \\
$p_{1}^{L} \quad$ Probability of becoming infected for a larva feeding on an infected \\
$q_{1}^{N} \quad$ host 1 \\
$\lambda_{L N}$ & Probability of becoming infected for a host 1 bitten by a nymph \\
$\lambda$ & Co-feeding probability between larvae and infected nymphs \\
$\alpha_{I}$ & Recovery rate for hosts 1 \\
\hline
\end{tabular}

\subsection{Effect of host densities on tick persistence and dynamics}

The relative densities of hosts that allow ticks to persist can be shown by a persistence-extinction boundary in the plane $H_{1}$ (rodents) - $H_{2}$ (deer); the curve $R_{0}=1$ that divides the region of host densities where tick population persists from the region in which ticks go extinct (Fig. 3). 


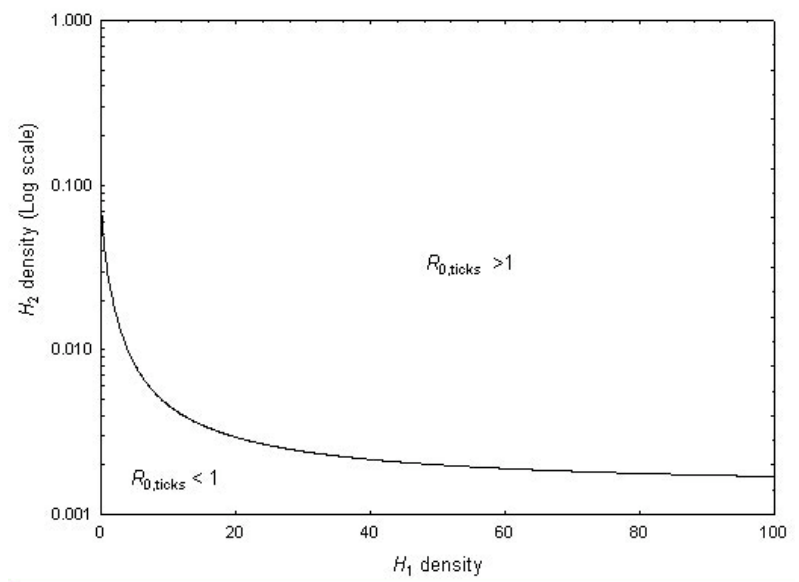

Fig. 3. The effect of host densities on the persistence of tick populations. The parameter values are: $\beta_{1}^{L}=0.03, \beta_{1}^{N}=0.001, \beta_{1}^{A}=0, \beta_{2}^{L}=0.05$, $\beta_{2}^{N}=0.05, \beta_{2}^{A}=0.28, d^{L}=d^{N}=d^{A}=0.02, r_{T}=1300$.

Tick populations persist as long as there are a few roe deer for the adult ticks to complete their life cycle. The ticks can persist with very low densities of rodents as long as this is compensated for by some increase in deer density. Note that the curve $R_{0}=1$ in Fig. 3 does not cross the rodent axis because we assume no adult ticks feed on rodents $\left(\beta_{1}^{A}=0\right)$.

The basic reproductive number is usually defined assuming there are no density-dependent constraints acting anywhere in the tick life cycle (Hudson et al. 2002). However, for model simulations it is important to introduce some density-dependent factor into tick life cycle to avoid unrealistic exponential tick population growth (or decrease). Norman et al. (1999) and Rosà et al. (2003b) assumed that density-dependence occurs in the production of larvae per feeding adult tick, indicated with the decreasing function $a_{T}(T)$. More precisely, they assumed that the production of larvae per feeding adult tick is a linear function of the total number of ticks present in the system:

$$
a_{T}(T)=r_{T}-s_{T} T \text {. }
$$

It may be more reasonable to assume that egg production depends on the average tick load of hosts, rather than on the absolute density of ticks. Since individual tick fecundity varies directly with meal size, a possible biological explanation for density dependence in tick fecundity is that hosts develop an immune response after being exposed to several tick bites (Wikel 1982; Randolph 1994; Hughes and Randolph 2001) which may de- 
crease the average blood meal taken by adult female ticks (Hudson and Dobson 1995). Without considering individual tick loads and immune histories, this mechanism can be modelled as adult fecundity depending on average tick load; using again a linear model the following form for the production of larvae per feeding adult is:

$$
a_{T}(T)=r_{T}-\mathrm{s}_{T} T /\left(u H_{1}+v H_{2}\right)
$$

The two parameters $u$ and $v$ are weights associated with the two host species that take into account their different contributions to tick population dynamics.

Fig. 4 shows the effect of host 1 density on tick equilibria using different choices for density-dependence in tick fecundity, $a_{T}(T)$. The same effect (not shown here) occurs for increasing density of host 2 . When hosts are abundant, ticks are more likely to attach to a host, hence there is an increase in the rates at which ticks progress from stage to stage and reproduce. However, as a result of negative density-dependence in fecundity, the reproductive success of ticks decreases with tick density, so that equilibrium density will saturate with increasing host density. Panel $A$ of Fig. 4 illustrates the case using the density-dependence function in (7). Under this assumption, it generally follows that the equilibrium density of larvae will depend in a non-monotonous way on the density of host 1 (the type on which larvae mainly feed). In fact, at high host densities, the total number of ticks at equilibrium is almost independent of host density; hence, the rate at which new larvae are recruited is practically constant. On the other hand, the rate at which larvae feed (and thus leave the stage) is still a strongly increasing function. An almost constant recruitment of questing larval ticks combined with a rate of removal that increases with host density must result in an equilibrium density of questing larvae that decreases with increasing density of host 1 .

Instead, if tick fecundity depends on average tick load $\left[a_{T}(T)\right.$ in (8)], the effect of host density on tick equilibrium density changes substantially. The resulting effect of host 1 density on equilibrium tick densities is shown in panel $B$ of Fig 4 . In this case, tick density increases almost linearly with host density without reaching a plateau as in the previous case, because ticks fecundity is regulated by tick:host ratio, so that ticks' carrying capacity increases almost linearly with host densities.

Density-dependence in ticks may also occur in different periods of its life cycle; for example, in Randolph and Rogers (1997), density dependence was detected in moulting probabilities. The same qualitative effect of host densities on tick equilibria was observed with model (5), considering 
density dependence in ticks moulting success (Rosà and Pugliese, submitted).
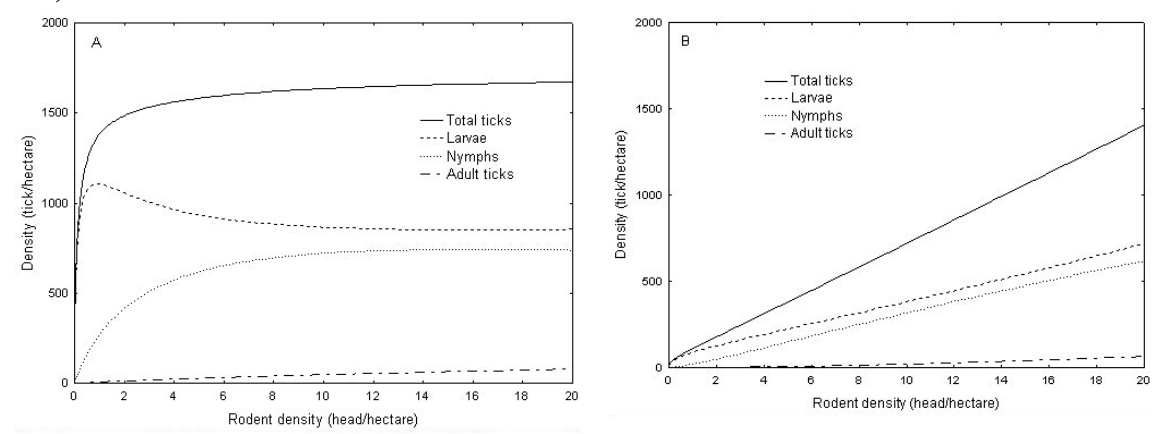

Fig. 4. Effect of rodent density on tick equilibrium densities. In panel $A a_{T}(T)$ in (7) is used while in panel $B$ we used $a_{T}(T)$ in (8). Parameter values are as in Fig. 3 ; the others are $m_{L}=m_{N}=0.2, \quad \sigma=0.25, r_{T}=1300, s_{T}=0.73, u=0.04, v=0.4$

\subsection{An example of a model for tick-borne infection}

Various tick-borne infections have different competent hosts, and diverse infection pathways. For instance, the dynamics of Lyme disease involves the spirochaete Borrelia burgdorferi s.l., the ticks that carry the bacteria and different hosts (rodents and deer). Some hosts, such as rodents, act as reservoirs of the infection, meaning that they can acquire the pathogen from infected ticks and transmit it to other ticks. Other hosts, like deer, are classified as tick maintenance hosts and they simply amplify the tick population without amplifying the pathogen. For Lyme disease, the main route of transmission is from an infected tick to a susceptible host and vice versa: this type of transmission is usually called systemic transmission. Recently it has been discovered (Gern and Rais 1996) that pathogens can be also transmitted from an infected tick to a non-infected tick while they co-feed on the same host: this process is known as non-systemic transmission. This co-feeding transmission seems to be very efficient for tick-borne encephalitis (TBE). Many workers have demonstrated that certain tick hosts, rodents in particular, which do not produce a viraemic response to TBE virus will permit non-viraemic transmission between co-feeding ticks (Jones et al. 1987; Labuda et al. 1993). Randolph et al. $(1996,1999)$ have shown the importance of co-feeding and temporal coincidence of different tick stages in the maintenance of TBE.

The classical method of deriving a model for tick-borne infection is to modify a tick population model by introducing the infection status of hosts 
and the various tick stages following the classical approach of SIR (susceptible, infected, recovered) models. Rosà and Pugliese (submitted), modifying model (5), have developed several tick-borne infection models that differ in their assumptions of tick dynamics, the competence of the various host species and different infection pathways occurring between hosts and ticks. Here we report some results obtained with one of those models that includes both systemic and non-systemic transmission, and considers two classes of hosts: hosts of type 1 acting both as reservoirs for the pathogens and as hosts for ticks, and hosts of type 2 which act only as feeding hosts for ticks. Questing and feeding tick stages are explicitly modelled and all stages are divided between susceptible and infected. Furthermore, hosts of class 1, are divided into susceptible, infective and immunes and their densities will change following infections and recoveries. No trans-ovarial transmission is assumed, hence questing larvae can only be susceptible, on the other hand, the pathogen is transmitted interstadially, so once an immature stage is infected, the subsequent stages can transmit the pathogen to a susceptible host (for the details of all processes included in the model see Rosà and Pugliese, submitted). This case appears adequate to describe the transmission of borreliosis or TBE, assuming host 1 to be small rodents, and host 2 to be mainly deer (Randolph et al. 2002).

The threshold condition for pathogen persistence found with the model is the following (Rosà and Pugliese, submitted):

$R_{0, \text { pathogen }}=\frac{m^{L} p_{1}^{L} \beta_{1}^{L} L_{Q}}{d_{1}+\gamma_{1}+\alpha_{1}} \frac{q_{1}^{N} \beta_{1}^{N} H_{1}}{d^{N}+\beta_{1}^{N} H_{1}+\beta_{2}^{N} H_{2}}+\frac{m^{L} \beta_{1}^{L}\left(\beta_{1}^{N} / \sigma^{N}\right) \lambda_{L N} L_{Q} H_{1}}{d^{N}+\beta_{1}^{N} H_{1}+\beta_{2}^{N} H_{2}}>1$

The expression for $R_{0}$ in (9) can be read as the expected number of infected larvae produced (in a wholly susceptible population) by a newly infected larva over its infectious period: the first term computes those infected through the systemic route (i.e. the probability of surviving, moulting, finding a host 1 and infecting it multiplied by the average number of larvae infected by that host); the second term computes those infected through the non-systemic route (i.e. the probability of surviving, moulting, finding a host 1 and infecting multiplied by times the average number of co-feeding larvae infected over that host). As for most macroparasite species (see sub-chapter 2) ticks are aggregated on hosts. In addition, the aggregated distributions of different tick stages are coincident rather than independent: those hosts feeding large number of larvae were simultaneously feeding the greatest number of nymphs (Craine et al. 1995; Randolph et al. 1996). It has been surmised that this pattern of tick infestation facilitates transmission via co-feeding and thus significantly increases the basic reproductive number $R_{0}$ of the pathogen (Randolph et al. 1999). 
To explore this, Rosà et al. (2003b) incorporated the effects of tick aggregation and correlation of different tick stages in the non-systemic route of transmission. They assumed that both immature tick stages distributions follow a negative binomial distribution and that larvae and nymphs are positively correlated on hosts. Specifically, they used the following expression for the co-feeding probability between larvae and infected nymphs:

$$
\lambda_{L N}=\theta_{L N}\left(1+\rho_{L N} / \sqrt{k^{L} k^{N}},\right.
$$

where $k^{L}$ and $k^{N}$ are the negative binomial aggregation parameters for larvae and nymphs, respectively, while $\rho_{L N}$ represents the correlation coefficient between larvae and nymphs (see Rosà et al. 2003b for details).

As for ticks, we focus on the effect of host densities on the persistence of the pathogen. In Fig. 5 the curves $R_{0}=1$ divides the region of host densities where pathogen persists from the region in which the pathogen goes extinct. The curve with a solid line represents one possible output of the model with systemic transmission only, while the dashed line shows a simulation when non-systemic transmission is also included in the model (Fig. 5). Clearly, the insertion of an additional route of transmission causes an increase of $R_{0}$, and the region where pathogen persists becomes larger (Fig. 5). A higher level of aggregation in larvae and nymphs distributions (lower values of $k^{L}$ and $k^{N}$ ) and a stronger correlation between larvae and nymphs (higher values of $\rho_{L N}$ ) increase the co-feeding transmission between larvae and infected nymphs [see the expression of $\lambda_{L N}$ in (10)] with a consequent rise of the basic reproductive number of the pathogen (Fig. 5).

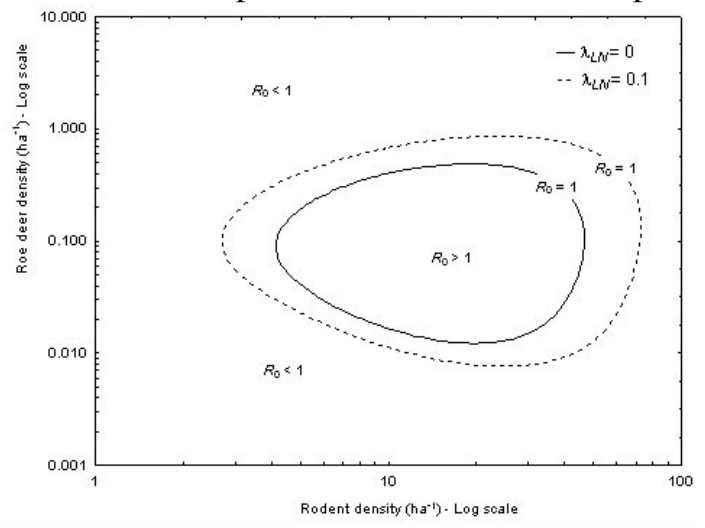

Fig. 5. Effect of host densities density on the $R_{0, \text { pathogen }}$ in (8) when only systemic infection is considered (solid line) an when non-systemic transmission is added (dashed line). Parameters values are as in Fig. 4 with $a_{T}(T)$ in (6); the others are $d=0.003, p_{1}^{L}=1, q_{1}^{N}=0.025, \gamma_{1}=0.01, \alpha_{1}=0.005$ 
The pathogen persists when $H_{2}$ density is in a range above a minimum density, needed for tick persistence, and below a maximum. Above the latter density, the incompetent hosts (roe deer) prevent the transmission of the disease, by acting as a pathogen sink that loses more pathogens from the system than the competent rodent hosts may produce. In other words, an increase in roe deer density has a positive effect on tick abundance but causes a dilution effect through 'wasted bites'; in this way, at high deer density, $R_{0}$ falls below unity (Fig. 5).

As for rodent density, both curves in Fig. 5 show that a minimum rodent density is needed for the pathogen to persist $\left(R_{0}>1\right)$. This is obvious, since infection is assumed to be transmitted only through rodents. The shape of the persistence curve also shows a dilution effect due to rodents: when rodent density is too high, the infection cannot persist. This is because for this simulation, the function $a_{T}(T)$ in (6) is used. In this case, ticks density (and especially larvae) does not increase much with increasing $H_{1}$ (Fig. 4 panel $A$ ), so that tick:host ratio will strongly decrease with increasing $H_{1}$. Thus, when hosts are abundant, each host will have the opportunity to infect only a few larvae; although each infected larva will then have a high probability of finding a host as a nymph, the overall effect is to decrease the reproduction ratio below 1 .

However, Rosà and Pugliese (submitted) showed that the negative effect of high rodent density strongly depends on the structure of densitydependence in ticks, and more generally, that tick population dynamics and its interaction with hosts plays a crucial role in the transmission of tick-borne pathogens. Hence, understanding how tick population dynamics depends on host densities and the biodiversity of the environment is a necessary step for drawing conclusions about the dynamics and persistence of tick-borne infections.

\section{Concluding remarks}

Recent years have seen a dramatic increase in the mathematical modelling of epidemics and an increasing recognition of the need to view such problems in their proper ecological context as host-parasite interactions. Experiments with infectious diseases in natural populations are often unethical, very expensive or impractical, and modelling provides the means of making explorative predictions.

One of the advantages of using epidemiological models is to develop explicit formulae for determining thresholds, equilibria, and periodic solutions and to provide a clear understanding of disease dynamics. Thus, 
models are also essential tools for identifying possible disease control strategies.

Throughout this chapter we focus on the threshold condition for parasite persistence, examining the effect of a specific aspect or variable, such as host densities and/or aggregation in parasite distribution, on parasite persistence. One important result is that the level of aggregation in tick distributions may influence the basic reproduction number of tick-borne infections, and consequently, the persistence of the disease. Not including tick aggregation among hosts on which non-systemic transmission takes place might cause an underestimate of $R_{0}$ of the infection, possibly leading to inappropriate conclusions.

This result emphasizes the importance of considering heterogeneities in the modelling of host-parasite interactions. Even more important is the modelling of biological mechanisms that produce aggregation in parasite distributions, rather than describing aggregation with particular population parameters, such as $k$ of the negative binomial distribution, which not correspond to any biological process, but are simply population statistics.

Some factors which may have a profound effect on infection transmission are missing in the models reviewed in this chapter, both for nematode and tick-borne infections, such as seasonality (White et al. 1996), multispecies and/or trophic levels (Grenfell 1992; Begon and Bowers 1995) and immunity (Woolhouse 1992; Grenfell et al. 1995). The insertion of these factors would certainly improve the models, although explicit expressions for thresholds and equilibria will probably no longer be computable.

On the whole, wildlife disease modelling remains an essential tool for understanding the increasing flood of data on hosts and pathogens. However, before using models as management tools for planning control and prevention programs, detailed empirical studies are needed to assess model results.

\section{References}

Abu-Madi MA, Behnke JM, Lewis JW, Gilbert FS (1998) Descriptive epidemiology of Heligmosomoides polygyrus in Apodemus sylvaticus from three contrasting habitats in south-east England. J Helmintol 72:93-100

Adler FR, Kretzschmar M (1992) Aggregation and stability in parasite-host models. Parasitol 104:199-205

Anderson RM, May RM (1978) Regulation and stability of host-parasite population interactions. I. Regulatory processes. J Anim Ecol 47:219-247

Anderson RM, May RM (1982) Coevolution of hosts and parasites. Parasitol $85: 411-426$ 
Anderson RM, May RM (1985) Age-related changes in the rate of transmission: implications for the design of vaccinations programmes. J Hygiene 94:365436

Anderson RC (2000) Nematode parasites of vertebrates: Their developement and transmission $2^{\text {nd }}$ edn. CABI Publ, Wallingford, Oxon

Arneberg P, Skorping A, Read AF (1998) Parasite abundance, body size, life histories and the energetic equivalent rule. Am Nat 151:497-513

Bailey NTJ (1975). The mathematical theory of infectious diseases and its applications. Griffin, London

Begon M, Bowers RG (1995) Beyond host-pathogen dynamics. In Grenfell BT, Dobson AP (eds) Ecology of infectious diseases in natural populations. Cambridge Univ Press, Cambridge, pp 478-509

Behnke JM, Keymer AE, Lewis JW (1991) Heligmosomoides polygyrus or Nematospiroides dubius? Parasitol Today 7:177-179.

Behnke JM, Lewis JW, Mohd Zain SN, Gilbert FS (1999) Helminth infections in Apodemus sylvaticus in southern England: Interactive effecys of host age, sex and year on the prevalence and abundance of infections. J Helmintol 73: 3144.

Cattadori IM, Boag B, Bjornstadt ON, Cornell SJ, Hudson PJ (2005) Peak shift and epidemiology in a seasonal host-nematode system. Proc R Soc Lond B 272:1163-1169.

Chan MS, Mutapi F, Woolhouse MEJ, Isham VS (2000) Stochastic simulation and the detection of immunity to schistosome infections. Parasitol 120:161-169

Cornell S (2005) Modelling nematodes populations: 20 years of progress. Trends Parasitol 11:483-546

Craine NG, Randolph SE, Nuttall PA (1995) Seasonal variation in the role of grey squirrels as hosts of Ixodes ricinus, the tick vector of the Lyme disease spirochaete, in a British woodland. Folia Parasitol 42:73-80

Crofton HD (1971) A quantitative approach to parasitism. Parasitol 63:179-193

Diekmann O, Kretzschmar M (1991) Patterns in the effects of infectious diseases on population growth. J Math Biol 29:539-570

Dobson AP, Hudson PJ (1992) Regulation and stability of a free-living hostparasite system: Trichostrongylus tenuis in red grouse. II. Population models. J Anim Ecol 61:487-498

Duerr HP, Dietz K, Eichner M (2003) On the interpretation of age-intensity profiles and dispersion patterns in parasitological surveys. Parasitol 126:87-101

Eisen L, Lane RS (2002) Vectors of Borrelia burgdorferi sensu lato. In: Gray O, Kahl RS, Lane RS, Stanek G (eds) Lyme borreliosis: Biology, epidemiology and control, CABI Publ, New York, pp 91-115

Enriquez FJ, Scarpino V, Cypress RH, Wassom DL (1988) In vivo and in vitro egg production by Nematospiroides dubius during primary and challenge infections in resistant and susceptible strains of mice. J Parasitol 74:262-266

Fernàndez S, Šarkunas M, Roepstorff A (2001) Survival of infective Ostertagia ostertagi larvae on pasture plots under different simulated grazing conditions. Vet Parasitol 96:291-299 
Ferrari N, Cattadori IM, Nespereira J, Rizzoli A., Hudson PJ (2004) The role of host sex in parasite dynamics: field experiments on the yellow-necked mouse Apodemus flavicollis. Ecol Letters 7:88-94.

Flowerdew J (1984) Wood mice. Anthony Nelson, Oswestry, Shropshire

Gaba S, Ginot V, Cabaret J (2005) Modelling macroparasite aggregation using a nematode-sheep system: The Weibull distribution as an alternative to the negative binomial distribution? Parasitol 131:393-401

Gern L, Rais O (1996) Efficient transmission of Borrelia burgdorferi between cofeeding Ixodes ricinus ticks (Acari:Ixodidae). J Med Entomol 33:189-192

Ghosh, M, Pugliese A (2004) Seasonal population dynamics of ticks, and its influence on infection transmission: A semi-discrete approach. Bull Math Biol 66:1659-168

Gilbert L, Norman R, Laurenson KM, Reid HW, Hudson PJ (2001). Disease persistence and apparent competition in a three-host community: an empirical and analytical study of large-scale, wild populations. J Anim Ecol 70:10531061

Gregory RD, Keymer AE, Clarke JR (1990) Genetics, sex, and exposure: the ecology of Heligmosomoides polygyrus (Nematoda) in the wood mouse. J Anim Ecol 59:363-378

Gregory RD (1991) Parasite epidemiology and host population growth: Heligmosomoides polygyrus (nematoda) in enclosed wood mouse populations. J Anim Ecol 60:805-821

Gregory, RD (1992) On the interpretation of host-parasite ecology: Heligmosomoides polygyrus (Nematoda) in wild wood mouse (Apodemus sylvaticus) populations. J Zool:226:109-121.

Gregory RD, Montgomery SSJ, Montgomery WI (1992) Population biology of Heligmosomoides polygyrus in the wood mouse. J Anim Ecol. 61:749-757.

Grenfell BT (1992) Parasitism and the dynamics of ungulate grazing systems. Amer Nat 139:907-929

Grenfell BT, Dietz K, Roberts MG (1995) Modelling the immuno-epidemiology of macroparasites in wildlife host populations In: Grenfell BT, Dobson AP (eds) Ecology of infectious diseases in natural populations. Cambridge Univ Press, Cambridge, pp 362-383

Herbert J, Isham V (2000) Stochastic host-parasite interaction models. J Math Biol 40:343-371

Hess G (1996) Disease in metapopulation models: Implications for conservation. Ecology 77:1617-1632

Hudson PJ, Dobson AP (1995) Macroparasites: Observed patterns. In: Grenfell BT, Dobson AP (eds) Ecology of infectious diseases in natural populations. Cambridge Univ Press, Cambridge, pp 144-176

Hudson PJ, Norman R, Laurenson MK, Newborn D, Gaunt M, Jones L, Reid H, Gould E, Bowers R, Dobson AP (1995) Persistence and transmission of tickborne viruses: Ixodes ricinus and louping-ill virus in red grouse populations. Parasitol 111:S49-S58

Hudson PJ, Dobson AP, Newborn D (1998). Prevention of population cycles by parasite removal. Science 282:2256-2258 
Hudson PJ, Rizzoli A, Grenfell BT, Heesterbeek H, Dobson AP (eds) (2002). The ecology of wildlife diseases. Oxford Univ Press, Oxford

Hughes VL, Randolph SE (2001) Testosterone depresses innate and acquired resistance to ticks in natural rodent hosts: a force for aggregated distributions of parasites. J Parasitol 87:49-54

Isham V (1995) Stochastic models of host-macroparasite interaction. Ann Appl Prob 5:720-740

Jones LD, Davies CR, Steele GM, Nuttall PA (1987) A novel mode of arbovirus transmission involving a nonviraemic host. Science 237:775-777

Jones LD, Gaunt M, Hails RS, Laurenson K, Hudson PJ, Reid H, Henbest P, Gould EA (1997) Transmission of louping-ill virus between infected and uninfected ticks co-feeding on mountain hares (Lepus timidus). Med Vet Entomol 11:172-176

Kaitala V, Ranta E Lindstroem J (1996) Cyclic population dynamics and random perturbations. J Anim Ecol 65:249-251

Keeling MJ (1999) The effects of local spatial structure on epidemiological invasions. Proc R Soc Lond B 266:859-867

Keymer AE (1985) Experimental epidemiology: Nematospiroides dubius and laboratory mouse. In: Rollison D, Anderson RM (eds) Ecology and genetics of host-Parasite interactions, Acad Press, London, pp.55-75

Keymer AE, Hiorns RW (1986) Heligmosomoides polygyrus (Nematoda): The dynamics of primary and repeated infection in outbred mice. Proc R Soc Lon B 229: 47-67

Kitron U, Mannelli A (1994) Modeling the ecological dynamics of tick-borne zoonoses. In: Mather TN, Sonenshine DE (eds) Ecological dynamics of tickborne zoonoses. Oxford Univ Press, Oxford, pp 198-239

Kostizin VA (1934) Symbiose, parasitisme et èvolution (ètude mathèmatique). Hermann, Paris. Translated in: Scudo F, Ziegler J (eds) The golden age of theoretical ecology. Lecture notes in biomathematics, Vol 52. SpringerVerlag, Berlin, 1978, pp 369-408

Labuda M, Jones LD, Williams T, Nuttal P (1993) Enhancement of tick borne encephalitis virus transmission by tick salivary gland extracts. Med Vet Entomol 7:193-196

Labuda M, Kozuch O, Zuffova E, Eleckova E, Hails RS, Nuttal PA (1997) Tickborne encephalitis virus transmission though ticks co-feeding on specific immune natural rodent hosts. Virology 235:138-143

Labuda M, Randolph SE (1999) Survival strategy of tick-borne encephalitis virus: cellular basis and environmental determinants. Zentralbl Bakteriol 289:513524

Lewis JW (1987) Helminth parasites of British rodents and insectivores. Mammal Rev 17:81-93

LoGiudice K, Ostfeld RS, Schmidt KA, Keesing F (2003) The ecology of infectious disease: Effects of host diversity and community composition on Lyme disease risk. Proc Natl Acad Sci USA 100:567-571

McCallum H, Dobson AP (1995) Detecting disease and parasite threats to endangered species and ecosystems. Trends Ecol Evol 10:190-194 
McCurdy DG, Shutler D, Mullie A, Forbes MR (1998) Sex-biased parasitism of avian host: Relations to blood parasite taxon and mating system. Oikos 82:303-312.

Moore SL, Wilson K (2002) Parasites as a viability cost of sexual selection in natural populations of mammals. Science 297:2015-2018

Mount GA, Haile DG (1987) Computer management of area-wide management strategies for the lone star tick, Ambllyomma americanum (Acari: Ixodidae) J Med Entomol 24:523-531

Mount GA, Haile DG (1989) Computer simulation of population dynamics of the America dog tick (Acari: Ixodidae). J Med Entomol 26:60-76

Norman R, Bowers RG, Begon M, Hudson PJ (1999) Persistence of tick-borne virus in the presence of multiple host species: tick reservoirs and parasite mediated competition. J Theor Biol 200:111-118

Ostfeld RS, Keesing F (2000) Biodiversity and disease risk: The case of Lyme disease. Conserv Biol 14:722-728

Perkins SE, Cattadori IM, Tagliapietra V, Rizzoli A, Hudson PJ (2003) Empirical evidence for key hosts in persistence of a tick-borne disease Int $\mathbf{J}$ Parasitol 33:909-917

Poulin R (1996) Sexual inequalities in helminth infections: A cost of being male? Amer Nat 147:287-295

Pugliese A (2002) Virulence evolution in macro-parasites. In: Castillo-Chavez C, Blower S, Kirschner D, Van Den Driessche P, Yakubu A (eds) Mathematical approaches for emerging and reemerging infectious aiseases: Models, methods and theory, Part 2. IMA Series vol. 126, SpringerVerlag, New York, pp 193-213

Pugliese A, Tonetto L (2004) Thresholds for macroparasite infections. J Math Biol 49:83-110

Quinnell RJ (1992) The population dynamics of Heligmosomoides polygyrus in an enclosure population of wood mice. J Anim Ecol 61:669-679

Quinnell RJ, Grafen A, Woolhouse MEJ (1995). Changes in parasite aggregation with age: A discrete infection model. Parasitol 111:635-644

Randolph SE (1994) Density-dependent acquired resistance in natural and unnatural hosts. Parasitol 79:141:156

Randolph SE (2001) The shifting landscape of tick-borne zoonoses: Tick-borne encephalitis and Lyme borreliosis in Europe. Phil Trans R Soc Lon B 356:1045-1056

Randolph SE, Rogers DJ (1997) A generic population model for the African tick Rhipicephalus appendiculatus. Parasitol 115:265-279

Randolph SE, Rogers DJ (2000) Fragile transmission cycles of tick-borne encephalitis virus may be disrupted by predicted climate change. Proc R Soc Lond B 267:1741-1744

Randolph SE, Gern L, Nuttal PA. (1996) Co-feeding ticks: epidemiological significance for tick borne pathogens transmission. Parasitol Today 12:472-479

Randolph SE, Miklisova D, Lysy J, Rogers DJ, Labuda M (1999) Incidence from coincidence: Patterns of tick infestations in rodents facilitate transmission of tick-borne encephalitis virus. Parasitol 118:177-186 
Randolph SE, Green RM, Peacey MF, Rogers DJ (2000) Seasonal synchrony: The key to the tick-borne pathogen transmission. Parasitol 121:15-23

Randolph SE, Chemini C, Furlanello C, Genchi C, Hails RA, Hudson PJ, Jones LD, Medley G, Norman R, Rizzoli AP, Smith G, Woolhouse MEJ (2002) The ecology of tick-borne infections in wildlife reservoirs. In: Hudson PJ, Rizzoli A, Grenfell BT, Hesterbeek H, Dobson A P (eds) The ecology of wildlife diseases. Oxford Univ Press, Oxford, pp119-138

Roberts MG, Heesterbeek JAP (1995) The dynamics of nematode infections of farmed ruminants. Parasitol 110:493-502

Roberts MG, Smith G, Grenfell BT (1995) Mathematical models for macroparasites of wildlife. In: Grenfell BT, Dobson AP (eds) Ecology of infectious diseases in natural populations. Cambridge Univ Press, Cambridge, pp 177-208

Rosà R, Pugliese A (2002) Aggregation, stability and oscillations in different models for host-macroparasite interactions. Theor Pop Biol 61:319-334

Rosà R, Pugliese A (submitted) Basic reproductive number for tick-borne infections under different modelling assumptions. Math Biosci

Rosà R, Rizzoli A, Pugliese A, Genchi C (2000) Managing chamois (Rupicapra rupicapra) populations: A model with macroparasites infection and host dynamics. Hystrix 11:103-114

Rosà R, Pugliese A, Villani A, Rizzoli A (2003a) Individual-based vs. deterministic models for macroparasites: host cycles and extinction. Theor Pop Biol 63:295-307

Rosà R, Pugliese A, Norman R, Hudson PJ (2003b) Thresholds for disease persistence in models for tick-borne infections including non-viraemic transmission, extended feeding and tick aggregation. J Theor Biol 224:359-376

Schalk G, Forbes MR (1997) Male biases in parasitism of mammals: effects of study type, host age and parasite taxon. Oikos 78:67-74

Scott ME (1987) Regulation of mouse colony abundance by Heligmosomoides polygyrus. Parasitol 95:111-124

Scott ME (1990) An experimental and theoretical study of the dynamics of a mouse-nematode (Heligmosomoides polygyrus) interactions. Parasitol 101:75-92.

Skorping A, Jensen KH (2004) Disease dynamics: All caused by males? Trends Ecol Evol 19:219-220

Slater AF, Keymer AE (1988) Epidemiology of Heligmosomoides polygyrus in mice: experiments on natural transmission. Parasitol 93:177-187

Van Buskirk J, Ostfeld RS (1995) Controlling Lyme disease by modifying the density and species composition of tick hosts. Ecol Appl 5:1133-1140

White KAJ, Grenfell BT, Hendry RJ, Lejeune O, Murray JD (1996) Effect of seasonal host reproduction on host-macroparasite dynamics. Math Biosci 137:79-99

Wikel SK (1982) Immune response to arthropods and their hosts. Ann Rev Entomol 27:21-48

Wilson K, Bjørnstad ON, Dobson AP, Merler S, Poglayen G, Randolph SE, Read AF, Skorping A (2002) Heterogeneities in macroparasite infections: Patterns and processes. In: Hudson PJ, Rizzoli A, Grenfell BT, Heesterbeek H, Dobson 
AP (eds) The ecology of wildlife disease, Oxford Univ Press, Oxford, pp. 644

Woolhouse MEJ (1992) A theoretical framework for the immunoepidemiology of helminth infection. Parasite Immunol 14:563-578

Woolhouse MEJ, Dye C, Etard JF, Smith T, Charlwood JD, Garnett GP, Hagan P, Hii JLK, Ndhlovu PD, Quinnel RJ, Watts CH, Chandiwana SK, Anderson RM (1997) Heterogeneities in the transmission of infectious agents; implications for the design of control programs. Proc Natl Acad Sci USA 94:338-342

Zeman P, Benes C (2004) A tick-borne encephalitis ceiling in Central Europe has moved upwards during the last 30 years: possible impact of global warming? Int J Med Microbiol 37:48-54 